\title{
A Micro/nano Structural Model for Wettability of Moth Wing Surface
}

\author{
Yan FANG, Gang SUN*, Jing-shi YIN, Wan-xing WANG and \\ Yu-qian WANG
}

School of Life Science, Changchun Normal University, Changchun 130032, China

${ }^{*}$ Corresponding author

Keywords: Microstructure, Model, Wettability, Moth, Biomaterial.

\begin{abstract}
The micro-morphology, wettability and chemical composition of the moth wing surfaces were investigated by a scanning electron microscope (SEM), a contact angle (CA) meter and a Fourier transform infrared spectrometer (FT-IR). The micro-morphological models for hydrophobicity on the wing surface were established on the basis of the Cassie-Baxter equation. The wetting mechanism was discussed from the perspective of biological coupling. The moth wing surfaces are composed of naturally hydrophobic material and display micro/nano hierarchical structures, including primary structure (micrometric scales), secondary structure (submicro longitudinal ridges and lateral bridges) and tertiary structure (nano stripes). The wing surfaces exhibit high hydrophobicity (CA $150 \sim 156^{\circ}$ ) and low adhesion (sliding angle $1 \sim 4^{\circ}$ ). The cooperative effect of material element and structural element contributes to the special wettability of the wing surface. In micro-dimension, the smaller the width and the bigger the spacing of the scale, the stronger the hydrophobicity of the wing surfaces. In nano-dimension, the smaller the height and the smaller the width and the bigger the spacing of the longitudinal ridge, the stronger the hydrophobicity of the wing surfaces. The micro/nano structural model provides CA predictions in good accord with the data measured. The result may bring inspiration for biomimetic design and preparation of smart interfacial materials and novel self-cleaning coatings.
\end{abstract}

\section{Introduction}

Wettability (e.g. hydrophobicity, hydrophilicity, oleophobicity, lipophilicity, adhesion, etc.) is one of the fundamental properties of a solid surface, which is basically determined by chemical composition (free energy) and microstructure (roughness) of the surface [1 3]. In the last few years, the interfacial materials with desirable performances have attracted great interest due to the wide variety of applications in industrial, biomedical, military and domestic fields [4]. After millions of years of natural selection, the creatures have evolved peculiar structures, forms and functions to adapt to the environment $[5,6]$. Insect is the unique flying invertebrate, which has the most species, the largest population and the widest distribution throughout the world. Insect wing has become a popular bio-template because of its excellent characteristics such as attractive iridescence, superhydrophobic property, and quick heat dissipation ability [7]. Up to now, the hydrophobicity models for bio-surfaces are still very limited. In this work, the micro-morphological models for hydrophobicity on the moth wing surface were established on the basis of the Cassie-Baxter equation. The wetting mechanisms were discussed from the perspective of biological coupling. This work can not only promote our understanding of the wetting phenomenon on bio-surfaces, but 
provide enlightenment for artificial manipulation of surface wettability and biomimetic preparation of smart functional materials, novel microfluidic devices and easy-cleaning coatings.

\section{Materials and Methods}

\section{Materials}

The moth specimens of ten species in Sphingidae were collected from June through September 2016 in Changchun City, Jilin City and Harbin City of northeast China, and identified by systematic taxonomy. The wings were cleaned, desiccated and flattened, then cut into $5 \mathrm{~mm} \times 5 \mathrm{~mm}$ pieces from discal cell (Fig. 1). The distilled water for CA and sliding angle (SA) measurements was purchased from Tianjin Pharmaceuticals Group Co. Ltd., China. The volume of water droplet was $5 \mu$ l.

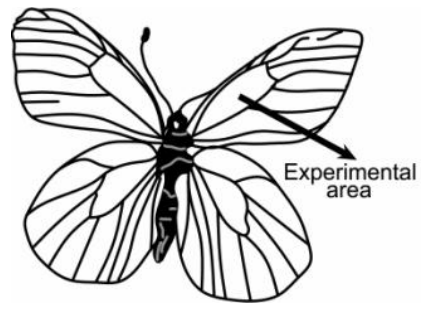

Figure 1. The experimental area of the wing surface

\section{Characterization of Micro/nano Structure}

After gold coating by an ion sputter coater (Hitachi E-1045, Japan), the wing pieces were observed and photographed by a SEM (Hitachi SU8010, Japan). Using Photoshop software, the microstructural parameters of the wing surfaces were measured in the SEM images.

\section{Measurements of Wetting Angles}

Using an optical CA measuring system (DataPhysics OCA20, Germany), the CA of water droplet on the wing surface was measured by sessile drop method at room conditions of $25 \pm 1{ }^{\circ} \mathrm{C}$ and relative humidity of approximately $80 \%$. The SA of water droplets was measured along three different directions, including forward SA (FSA, the SA of droplet from wing base to wing terminal end), backward SA (BSA, the SA of droplet from wing terminal end to wing base) and perpendicular SA (PSA, the SA of droplet perpendicular to the major axis of wing) (Fig. 1). The water droplet was dripped on the sample table in a horizontal position, then the inclination degree of the table was raised $1^{\circ}$ each time until the droplet rolled off freely. The inclination degree of the table was recorded as the SA value.

\section{FT-IR Measurement}

After grinding finely, 5 8 mg of wing samples were mixed homogeneously with 200 $\mathrm{mg}$ of $\mathrm{KBr}$ and pressed into a thin slice. The absorbance was measured by means of FT-IR (Nicolet FT-IR200, USA). The chemical composition of the wing surface was analyzed by the FT-IR spectra. 


\section{Data Treatment}

Each micro-morphological parameter and wetting angle (CA, SA) was measured five times for every moth species. Using SPSS software, linear regression analysis and significance test of difference were conducted.

\section{Results and Discussion}

\section{Micro-morphology of the Moth Wing Surface}

The moth wing surface exhibits multiple-dimensional rough structures. The micrometric scales constitute the primary structure [Fig. 2(A)]. The length ( $a$ in Table 1) of the scale is $183 \sim 345 \mu \mathrm{m}$, the width ( $b$ in Table 1) is $67 \sim 108 \mu \mathrm{m}$, the spacing ( $c$ in Table 1) is $92 \sim 143 \mu \mathrm{m}$. The submicro longitudinal ridges and lateral bridges on the scales constitute the secondary structure [Fig. 2(B)]. The longitudinal ridges and lateral bridges are linked as grids, some longitudinal ridges have branches. The height ( $d$ in Table 1) of the longitudinal ridge is $572 \sim 869 \mathrm{~nm}$, the width ( $e$ in Table 1) is 465 984 $\mathrm{nm}$, the spacing $(f$ in Table 1$)$ is $1.31 \sim 2.26 \mu \mathrm{m}$. The nano stripes distributing regularly on the longitudinal ridges and lateral bridges constitute the tertiary structure [Fig. 2(C)]. The cross-section of the longitudinal ridge is triangular [Fig. 2(D)].

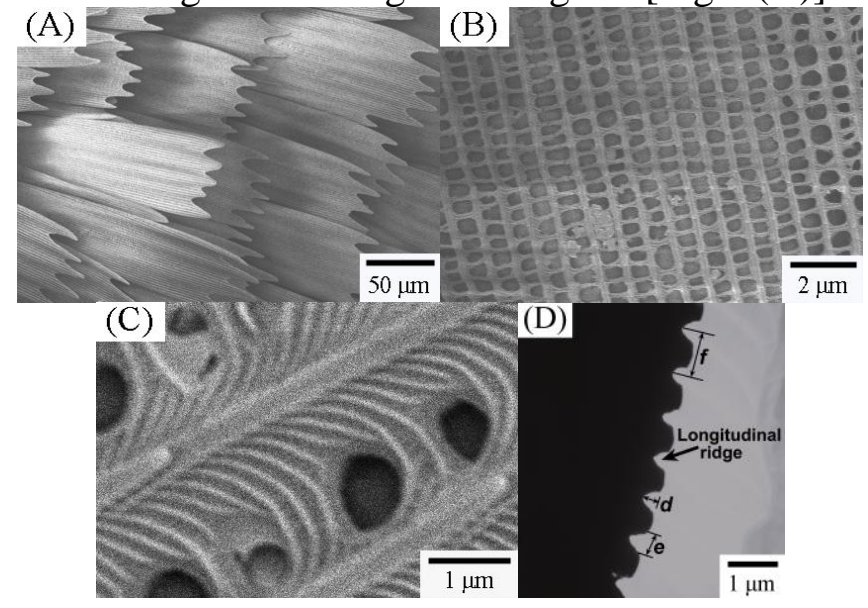

Figure 2. The multiple-dimensional rough micro-morphology of the moth wing surface

(A) Primary structure (micrometric scales); (B) Secondary structure (submicro longitudinal ridges and lateral bridges); (C) Tertiary structure (nano stripes); (D) Illustration of the structural parameters of scale.

\section{Superhydrophobicity and Low Adhesion on the Moth Wing Surface}

The moth wing surface is of low adhesive superhydrophobicity. The range of CA is $150 \sim 156^{\circ}$, the average of CA is $153^{\circ}$. The range of FSA is $1 \sim 4^{\circ}$, BSA $6 \sim 11^{\circ}$, PSA $4 \sim 8^{\circ}$ (Table 1). The large CA and small SA imply excellent self-cleaning property of the wing surface. Interestingly, there are extremely significant differences between FSA and BSA $(p<0.01)$, between FSA and PSA $(p<0.01)$, as well as between BSA and PSA $(p<0.05)$. The sliding behavior of droplet on the moth wing surface displays remarkable anisotropism, which is the result of the oriented micro-morphology and the energy barrier difference in various directions. Such a special complex wettability on the moth wing surface resembles that on the butterfly wing surface [8]. 
Table 1. Microstructural parameters and wettability of the moth wing surfaces

\begin{tabular}{|c|c|c|c|c|c|c|c|c|c|c|c|c|c|}
\hline \multirow{2}{*}{ Species } & \multicolumn{3}{|c|}{ Scale $(\mu \mathrm{m})$} & \multicolumn{3}{|c|}{$\begin{array}{c}\text { Longitudinal } \\
\text { ridge }(\mu \mathrm{m})\end{array}$} & \multirow{2}{*}{$\begin{array}{c}\text { Measured } \\
\mathrm{CA}\left({ }^{\circ}\right)\end{array}$} & \multicolumn{3}{|c|}{ Predicted CA $\left({ }^{\circ}\right)$} & \multicolumn{3}{|c|}{$\begin{array}{c}\text { Measured SA } \\
\left({ }^{\circ}\right)\end{array}$} \\
\hline & $a$ & $b$ & $c$ & $d$ & $e$ & $f$ & & Model 1 & Model 2 & Model 3 & FSA & $\begin{array}{r}\mathrm{BS} \\
\mathrm{A} \\
\end{array}$ & PSA \\
\hline Amorpha amurensis & 224 & 85 & 126 & $\begin{array}{r}0.6 \\
5 \\
\end{array}$ & $\begin{array}{r}0.7 \\
3 \\
\end{array}$ & \begin{tabular}{r|}
1.7 \\
8 \\
\end{tabular} & 154 & 121 & 141 & 155 & 2 & \begin{tabular}{l|}
1 \\
1 \\
\end{tabular} & 6 \\
\hline $\begin{array}{c}\text { Ampelophaga } \\
\text { rubiginosa }\end{array}$ & 258 & 94 & 130 & $\begin{array}{r}0.7 \\
4 \\
\end{array}$ & \begin{tabular}{|r|}
0.7 \\
0 \\
\end{tabular} & \begin{tabular}{|r|}
1.3 \\
1 \\
\end{tabular} & 152 & 119 & 138 & 154 & 2 & 6 & 8 \\
\hline Callambulyx tatarinovi & 196 & 78 & 98 & \begin{tabular}{r|}
0.8 \\
7 \\
\end{tabular} & \begin{tabular}{|r|}
0.9 \\
2 \\
\end{tabular} & \begin{tabular}{r|}
2.1 \\
4 \\
\end{tabular} & 151 & 116 & 140 & 153 & 3 & 8 & 4 \\
\hline Dolbina exacta & 183 & 76 & 141 & $\begin{array}{r}0.6 \\
2 \\
\end{array}$ & \begin{tabular}{|r|}
0.4 \\
7 \\
\end{tabular} & $\begin{array}{r}2.0 \\
5 \\
\end{array}$ & 150 & 120 & 137 & 152 & 4 & 7 & 7 \\
\hline Dolbina tancrei & 312 & 67 & 93 & $\begin{array}{r}0.6 \\
5 \\
\end{array}$ & \begin{tabular}{|r|}
0.6 \\
7 \\
\end{tabular} & $\begin{array}{r}1.9 \\
5 \\
\end{array}$ & 156 & 116 & 140 & 158 & 2 & $\begin{array}{l}1 \\
0 \\
\end{array}$ & 8 \\
\hline Hyloicus caligineus & 249 & 101 & 106 & $\begin{array}{r}0.7 \\
8 \\
\end{array}$ & \begin{tabular}{|r|}
0.9 \\
8 \\
\end{tabular} & \begin{tabular}{r|}
1.8 \\
3 \\
\end{tabular} & 151 & 115 & 142 & 154 & 2 & 7 & 5 \\
\hline $\begin{array}{c}\text { Macroglossum } \\
\text { stellatarum }\end{array}$ & 236 & 93 & 143 & $\begin{array}{r}0.5 \\
7 \\
\end{array}$ & \begin{tabular}{|r|}
0.7 \\
8 \\
\end{tabular} & $\begin{array}{r}2.2 \\
6 \\
\end{array}$ & 152 & 118 & 133 & 150 & 2 & 8 & 5 \\
\hline Marumba jankowskii & 345 & 82 & 117 & \begin{tabular}{r|}
0.8 \\
3 \\
\end{tabular} & \begin{tabular}{|r|}
0.8 \\
6 \\
\end{tabular} & \begin{tabular}{r|}
1.8 \\
4 \\
\end{tabular} & 154 & 120 & 138 & 151 & 1 & 8 & 6 \\
\hline Pergesa elpenor & 307 & 108 & 128 & $\begin{array}{r}0.5 \\
9 \\
\end{array}$ & \begin{tabular}{r|}
0.8 \\
1 \\
\end{tabular} & $\begin{array}{r}1.8 \\
6 \\
\end{array}$ & 155 & 119 & 142 & 153 & 1 & 6 & 5 \\
\hline Sphecodina caudata & 264 & 86 & 123 & $\begin{array}{r}0.6 \\
1 \\
\end{array}$ & $\begin{array}{r}0.5 \\
6 \\
\end{array}$ & $\begin{array}{r}1.6 \\
4 \\
\end{array}$ & 151 & 118 & 140 & 153 & 3 & 9 & 7 \\
\hline Average & 257 & 87 & 121 & $\begin{array}{r}0.6 \\
9\end{array}$ & $\begin{array}{r}0.7 \\
5\end{array}$ & $\begin{array}{r}1.8 \\
7\end{array}$ & 153 & 118 & 139 & 257 & 2 & 8 & 6 \\
\hline
\end{tabular}

\section{Hydrophobicity Models of the Moth Wing Surfaces}

According to the FT-IR spectra (Fig. 3), the moth wing surfaces are composed of naturally hydrophobic material, such as chitin, protein, fat [9].

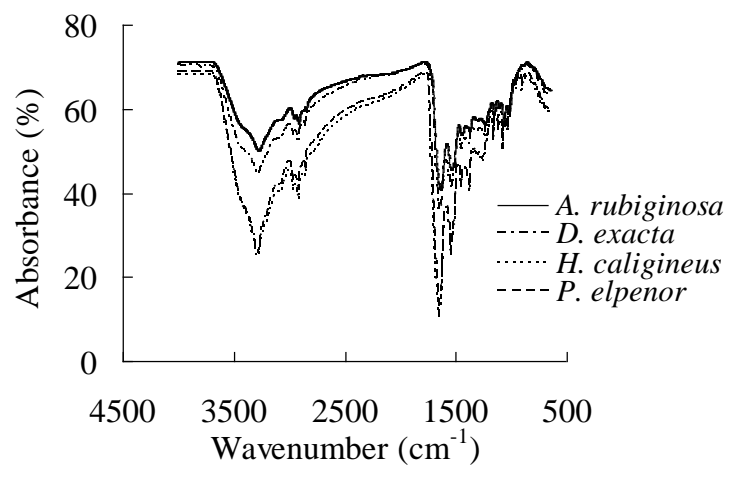

Figure 3. FT-IR spectra of the moth wing surfaces

The moth wing surface is relatively rough with superhydrophobicity and heterogeneity. A composite contact is formed between the droplet and the surface. Thus, the contact behavior of water droplet on the wing surface can be expressed by the following Cassie-Baxter equation:

$$
\cos \theta_{c}=\phi_{s} \cos \theta_{e}+\phi_{s}-1
$$

where $\theta_{c}$ is the apparent CA of a droplet on a heterogeneous composite surface, $\phi_{s}$ is the area fraction of solid on a composite surface $\left(0<\phi_{S}<1\right.$, calculated from microstructural parameters of the wing surface), $\theta_{e}$ is the intrinsic CA of a droplet on an ideal flat surface (approximately $95^{\circ}$ on the moth wing surface). 
Model I: sole effect of micrometric structure. It is assumed that scales with identical form and size align regularly; scale surfaces are smooth, without nano structure. The contact state of water droplet on the micrometric structure is shown in Fig. 4(A).

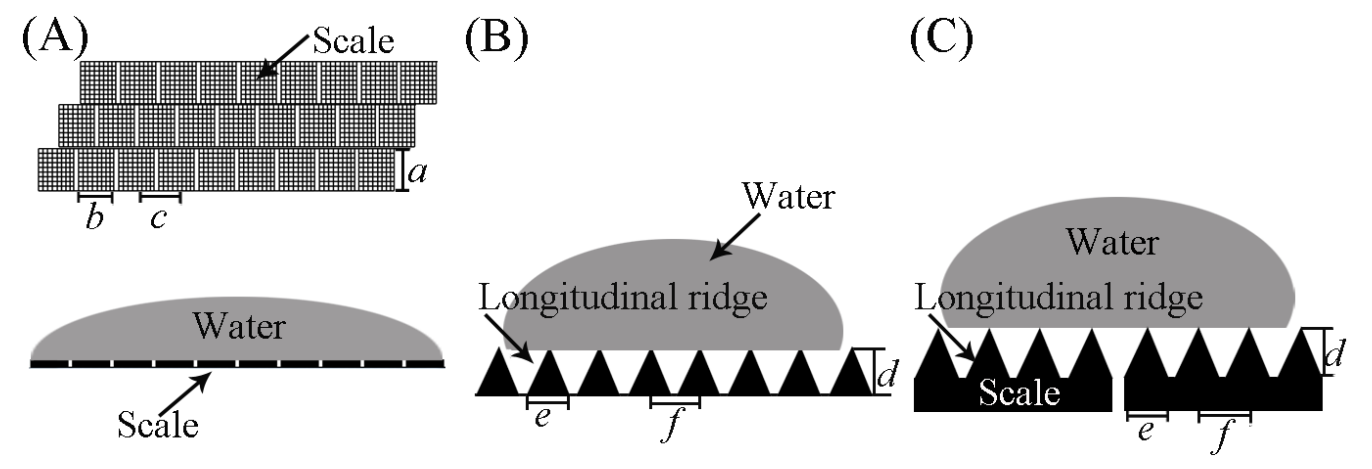

Figure 4. The contact states of water droplet on the moth wing surfaces

(A) Micrometric structure; (B) Nano structure; (C) Micro/nano structure.

In this case, the apparent $\mathrm{CA}\left(\theta_{m}\right)$ can be given as follows:

$$
\cos \theta_{m}=\frac{b}{c} * \cos \theta_{e}+\frac{b}{c}-1
$$

Model II: sole effect of nano structure. It is assumed that nano longitudinal ridges align regularly on scale; the cross-section of longitudinal ridge is triangular. The contact state of water droplet on the nano structure is shown in Fig. 4(B). In this case, Eq. (1) can be modified for the apparent $\mathrm{CA}\left(\theta_{n}\right)$ as follows:

$$
\cos \theta_{n}=\sqrt{\frac{4 d^{2}}{e^{2}}+1} * \frac{e}{f} * \cos \theta_{e}+\frac{e}{f}-1
$$

Model III: synergetic effect of micro/nano structure. It is assumed that scales with identical form and size align regularly; nano longitudinal ridges align regularly on scale; the cross-section of longitudinal ridge is triangular. The contact state of water droplet on the micro/nano structure is shown in Fig. 4(C). In this case, Eq. (1) can be modified for the apparent $\mathrm{CA}\left(\theta_{m n}\right)$ as follows:

$$
\cos \theta_{m n}=\sqrt{\frac{4 d^{2}}{e^{2}}+1} * \frac{b e}{c f} * \cos \theta_{e}+\frac{b e}{c f}-1
$$

Based on Eqs. (2) (4), the predicted CAs were calculated (Table 1). Taking predicted $\mathrm{CA}$ as an independent variable, measured CA as a dependent variable, simple linear regression analysis was conducted (Table 2).

Table 2. The results of linear regression analysis for the three hydrophobicity models

\begin{tabular}{|l|c|c|c|c|}
\hline \multicolumn{1}{|c|}{ Model } & $F$ & $p$ & $R^{2}$ & Adjusted $R^{2}$ \\
\hline Model I: sole effect of micrometric structure & 4.412 & 0.061 & 0.135 & 0.112 \\
\hline Model II: sole effect of nano structure & 29.536 & 0.000 & 0.462 & 0.448 \\
\hline Model III: synergetic effect of micro/nano structure & 45.824 & 0.000 & 0.653 & 0.634 \\
\hline
\end{tabular}

According to the result of significance test ( $F$ test), Model I (sole effect of micrometric structure) is unacceptable $(p>0.05)$, while Model II (sole effect of nano structure) and Model III (synergetic effect of micro/nano structure) have remarkable significance $(p<0.01)$. Using $R^{2}$ and adjusted $R^{2}$, Model 2 and Model 3 were further compared. Model 3 is superior to Model 2. This is in agreement with the micro-morphology of the moth wing surface, which is neither a one-dimensional micrometric structure nor a nano structure, but a multiple-dimensional hierarchical 
micro/nano structure. In the light of Model 3, the smaller the values of $\sqrt{\frac{4 d^{2}}{e^{2}}+1}$ and $\frac{b e}{c f}$, the bigger the theoretical CAs. Namely, smaller width and bigger spacing of the micrometric scale, as well as smaller height, smaller width and bigger spacing of the nano longitudinal ridge can lead to stronger hydrophobicity of the wing surfaces. Naturally hydrophobic material such as chitin is the chemical foundation for the superhydrophobicity on the moth wing surfaces. Much higher hydrophobicity, however, cannot be induced by the chemical composition alone. Superhydrophobicity of the wing surfaces is attributed to a combination of material element and structure element. Here, only the primary and secondary microstructures of the wing surfaces were incorporated in the hydrophobicity models. In fact, the more subtle tertiary microstructure (nano stripes) also contributes to the surface roughness and hydrophobicity. The hydrophobicity model involving tertiary microstructure is likely to offer more accurate CA predictions.

\section{Summary}

The moth wing surfaces are composed of naturally hydrophobic material and possess multiple-dimensional rough structures, including primary structure, secondary structure and tertiary structure. The wing surfaces exhibit high hydrophobicity (CA 150 156 ${ }^{\circ}$ ) and low adhesion (sliding angle $1 \sim 4^{\circ}$ ). The CA values predicted by the micro/nano structural model are in good accord with the CA values measured. In micro-dimension, the smaller the width and the bigger the spacing of the scale, the stronger the hydrophobicity of the wing surfaces. In nano-dimension, the smaller the height and the smaller the width and the bigger the spacing of the longitudinal ridge, the stronger the hydrophobicity of the wing surfaces. The cooperative effect of material element and structural element contributes to the special wettability of the wing surface. The moth wing can be used as a bio-template for design of micro-controllable superhydrophobic surface and self-cleaning coatings. This work may promote our understanding of wetting phenomenon on bio-surfaces, and bring inspiration for biomimetic preparation of smart interfacial material and novel microfluidic device.

\section{Acknowledgement}

This work was financially supported by the National Natural Science Foundation of China (Grant No. 31671010), the Natural Science Foundation of Jilin Province, China (Grant No. 201115162) and the Innovative Program for Postgraduate Students of Changchun Normal University (Grant No. cscxy2015007, cscxy2017003, cscxy2017006). Dr. Prof. Gang Sun is the corresponding author of this paper.

\section{References}

[1] T.L. Sun, L. Feng, X.F. Gao, L. Jiang, Bioinspired surfaces with special wettability, Accounts Chem. Res. 38 (2005) 644-652.

[2] X. Yao, Y.L. Song, L. Jiang, Applications of bio-inspired special wettable surfaces, Adv. Mater. 23 (2011) 719-734.

[3] S.T. Wang, L. Jiang, Definition of superhydrophobic states, Adv. Mater. 19 (2007) 3423-3424. 
[4] A.M. Higgins, R.A.L. Jones, Anisotropic spinodal dewetting as a route to self-assembly of patterned surfaces, Nature 404 (2000) 476-478.

[5] M. Gleiche, L.F. Chi, H. Fuchs, Nanoscopic channel lattices with controlled anisotropic wetting, Nature 403 (2000) 173-175.

[6] M.J. Liu, Y.M. Zheng, J. Zhai, L. Jiang, Bioinspired super-antiwetting interfaces with special liquid-solid adhesion, Accounts of Chemical Research, 43 (2010) 368-377.

[7] Y. Fang, G. Sun, Q. Cong, G.H. Chen, L.Q. Ren, Effects of methanol on wettability of the non-smooth surface on butterfly wing, J. Bion. Eng. 5 (2008) 127-133.

[8] Y. Fang, G. Sun, T.Q. Wang, Q. Cong, Hydrophobicity mechanism of non-smooth pattern on surface of butterfly wing, Chin. Sci. Bull. 52 (2007) 711-716.

[9] X.J. Wang, Q. Cong, J.J. Zhang, Y.L. Wan, Multivariate coupling mechanism of NOCTUIDAE moth wings' surface superhydrophobicity, Chin. Sci. Bull. 54 (2009) 569-575. 